\title{
The Inferior Parietal Lobule and Recognition Memory: Expectancy Violation or Successful Retrieval?
}

\author{
Akira R. 0'Connor, ${ }^{1}$ Sanghoon Han, ${ }^{2}$ and Ian G. Dobbins ${ }^{1}$ \\ ${ }^{1}$ Department of Psychology, Washington University in St. Louis, St. Louis, Missouri 63130, and ²Department of Psychology, Yonsei University, Seoul 120-749, Korea
}

Functional neuroimaging studies of episodic recognition demonstrate an increased lateral parietal response for studied versus new materials, often termed a retrieval success effect. Using a novel memory analog of attentional cueing, we manipulated the correspondence between anticipated and actual recognition evidence by presenting valid or invalid anticipatory cues (e.g., "likely old") before recognition judgments. Although a superior parietal region demonstrated the retrieval success pattern, a larger inferior parietal lobule (IPL) region tracked the validity of the memory cueing (invalid cueing $>$ valid cueing) and no retrieval success-sensitive lateral parietal region was insensitive to cueing. The invalid cueing response occurred even for correctly identified new items unlikely to trigger substantive episodic retrieval. Within the IPL, although supramarginal and angular gyrus (SMG; AG) regions both demonstrated invalid cueing amplitude elevations, each region differentially coupled with distinct cortical networks when unexpectedly old items were encountered; a connectivity pattern also observed at rest in the same subjects. These findings jointly suggest that the lateral parietal response during recognition does not signify the recovery of episodic content, but is a marker of the violation of memory expectations. A second independent dataset confirmed this interpretation by demonstrating that SMG activation tracked the decision biases of observers, not their accuracy, with increased activation for nondominant recognition judgments. The expectancy violation interpretation of the lateral parietal recognition response is consistent with the literature on visual search and oddball paradigms and suggests that damage to these regions should impair memory-linked orienting behavior and not retrieval per se.

\section{Introduction}

One of the most robust findings in functional neuroimaging studies of episodic memory is termed the "retrieval success" effect, which implicates an extensive network of prefrontal and parietal regions demonstrating greater activation for hits versus correct rejections (Konishi et al., 2000; McDermott et al., 2000; Wagner et al., 2005; Vilberg and Rugg, 2008). Recently, the left parietal response has generated considerable interest because of its correspondence to an event-related potential (ERP) component also thought to signify successful episodic recollection (Rugg and Doyle, 1992; Allan and Rugg, 1997; Wilding, 2000). In support of a primary role in recollection, fMRI activation in the angular gyrus (AG) region tracks subjective reports of recollection of prior experiences, increases as a function of the amount of episodic detail recovered, and is greater for source recognition than simple item recognition (Henson et al., 1999; Dobbins et al., 2003; Yonelinas et al., 2005; Daselaar et al., 2006). Additionally, analyses of the patterns of interregional covariance in BOLD activity at rest demonstrate robust connectivity between AG and hippocampus, further suggesting a direct role in memory retrieval (Vincent et al., 2006, 2008).

Despite this converging evidence, a direct role for lateral parietal cortex in memory retrieval remains questionable because

Received Aug. 26, 2009; revised Jan. 7, 2010; accepted Jan. 8, 2010.

This work was supported by National Institutes of Health Grant MH073982.

Correspondence should be addressed to Akira R. O'Connor, Department of Psychology, Washington University in

St. Louis, One Brookings Drive, Campus Box 1125, St. Louis, M0 63130. E-mail: aoconnor@wustl.edu.

DOI:10.1523/JNEUROSCI.4225-09.2010

Copyright $\odot 2010$ the authors $\quad 0270-6474 / 10 / 302924-11 \$ 15.00 / 0$ neuropsychological findings do not demonstrate prominent memory impairment associated with parietal lesions (e.g., Davidson et al., 2008; Simons et al., 2008). Instead, parietal patients appear to have preserved accuracy, but somewhat reduced confidence in memory judgments (Simons et al., 2010). This finding is perhaps more consistent with an alternative attention-based account of the lateral parietal response (Cabeza et al., 2008) adapted from the visual search and attention literature (Corbetta and Shulman, 2002; Corbetta et al., 2008). Under this model, the AG response is hypothesized to reflect attentional capture by evoked recollections. In contrast, the more superior supramarginal or intraparietal sulcus (IPS) response is argued to reflect a deliberate engagement in memory search. Within this framework the reduced confidence of patients' reports may reflect the reduced salience of probe-evoked memory events. Critically however, direct comparisons of visual attention and retrieval success responses demonstrate that the effects occur in separate but proximal superior and inferior parietal regions (Shannon and Buckner, 2004; Hutchinson et al., 2009). Thus any functional correspondence between visual and memory orienting mechanisms would have to reflect common principles that operate in different areas of cortex dedicated to different (visual vs mnemonic) representations.

Using event-related fMRI, we directly tested an attentional model of the lateral parietal recognition response using a paradigm that modulates expectations about upcoming memoranda during episodic recognition. We used an episodic analog of the Posner visual cueing task (Posner et al., 1980), providing participants with anticipatory mnemonic cues regarding upcoming test 


\begin{tabular}{|c|c|c|}
\hline \multicolumn{3}{|l|}{ A } \\
\hline \multicolumn{3}{|c|}{ session order } \\
\hline \multicolumn{3}{|c|}{ 1. high run validity (1) } \\
\hline \multicolumn{3}{|c|}{ 2. low run validity (1) } \\
\hline \multicolumn{3}{|c|}{ 3. high run validity (2) } \\
\hline \multicolumn{3}{|c|}{ 4. Iow run validity (2) } \\
\hline \multicolumn{3}{|l|}{ B } \\
\hline \multicolumn{3}{|c|}{ run details } \\
\hline run validity & high & low \\
\hline sessions & 1,3 & 2,4 \\
\hline $\begin{array}{l}\text { test trials } \\
\text { (per session) }\end{array}$ & 80 & 108 \\
\hline $\begin{array}{l}\text { fixation trials } \\
\text { (per session) }\end{array}$ & 20 & 18 \\
\hline valid cues & $80 \%$ & chance \\
\hline $\begin{array}{l}\text { neutral cues } \\
\text { (per session) }\end{array}$ & no & $\begin{array}{l}\text { yes } \\
(36)\end{array}$ \\
\hline feedback & yes & no \\
\hline
\end{tabular}

C

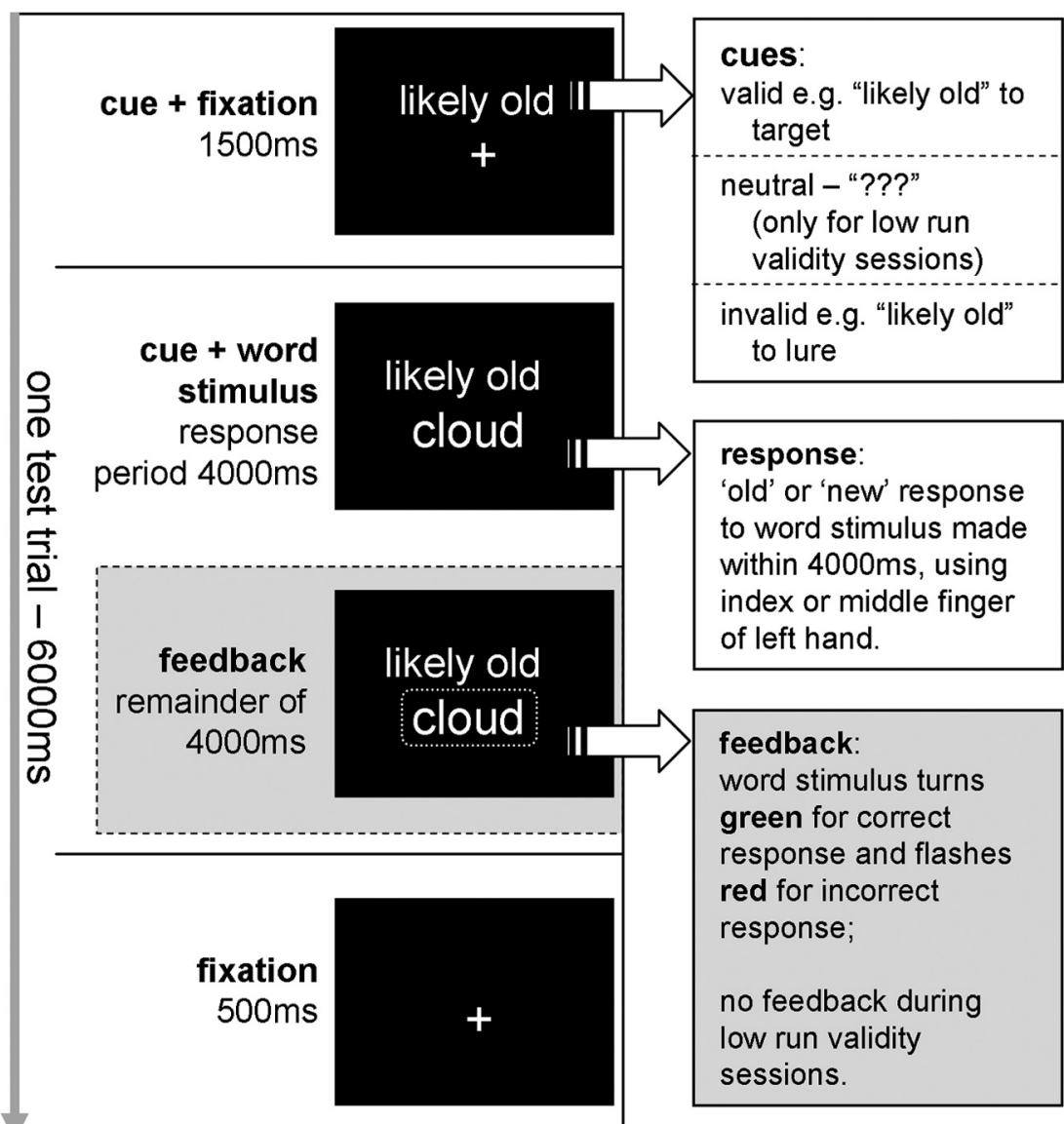

Figure 1. Schematic diagram of the experimental paradigm. $\boldsymbol{A}$, Test session order for all participants. B, Differences between low-run-validity sessions and high-run-validity sessions. $\boldsymbol{C}$, The components of a single $6000 \mathrm{~ms}$ test trial.

stimuli (e.g., "likely old") to manipulate whether expectations were confirmed or violated independently of item study status (target or lure). In the same participants, we measured restingstate functional connectivity to test the correspondence between task-evoked responses and resting-state connectivity. If parietal responses reflect mnemonic expectancy mechanisms rather than the successful recovery of episodic content, then they should demonstrate expectancy-linked responses regardless of item memory status, analogous to the manner in which parietal responses signal violations of visuospatial expectations during visual search.

\section{Materials and Methods}

Participants. Nineteen right-handed participants (20-32 years old, 11 women) were included, with data from five additional participants excluded due to technical difficulties during fMRI data acquisition, failure to understand the task or failure to complete all experimental runs. Informed consent was obtained in a manner approved by the Institutional Review Board of Washington University in St. Louis.

Stimuli and procedures. The experimental procedure used an episodic memory analog of the Posner cueing paradigm (Posner et al., 1980). In a series of four study-test cycles, participants first incidentally encoded words via syllable judgments ("1," "2," " 3 ," " 4 or more") with a scanned recognition test immediately following each study list. Critically, each test item was preceded by either a potentially informative cue indicating a possible status of the upcoming memory probe ("likely old" or "unlikely old"), or a neutral cue (“???"). During study-test cycles 1 and 3 (high run validity), the cues were frequently valid $(80 \%)$, participants received feedback immediately following each response, and neutral cues were not used. In each of these cycles, participants studied 40 items and judged 80 items at test (the 40 studied items and 40 new items). During cycles 2 and 4 (low run validity), the cues became random, neutral cues were also presented, and feedback was omitted. In these cycles, participants studied 54 items and judged 108 items at test. Participants were not informed of the cue validity probabilities. Therefore the alternating cycle procedure used feedback to instill confidence in the utility of cues in cycles 1 and 3, and examine the effects of cue-probe integration without potential contamination from feedback-linked processing in cycles 2 and 4. See Figure 1 for a summary of the design.

During structural scans, participants completed a short practice version of the experimental task in a format similar to cycles 1 and 3 . The order of conditions at test was determined by a genetic algorithm that optimized the design efficiency for the contrasts of interest (Wager and Nichols, 2003). For each participant, a different set of words was randomly sampled from a source list of 1216 nouns with a mean of 7.09 letters, 2.34 syllables, and mean Kucera-Francis frequency of 8.84 .

fMRI acquisition and preprocessing. Scanning was performed on a 3T Siemens Trio whole-body MRI scanner (Siemens Medical Solutions) using a standard whole-head coil. Functional data were acquired using an interleaved ascending echo-planar pulse sequence $(\mathrm{TR}=2000 \mathrm{~ms}, \mathrm{TE}=$ $27 \mathrm{~ms}, 34$ axial slices parallel to the AC-PC plane with isotropic $4 \mathrm{~mm}$ voxels, no interslice gap). The first two volumes were discarded to allow for $\mathrm{T} 1$ equilibration. Head motion was minimized using foam padding. High-resolution T1-weighted and T2-weighted anatomical images were also acquired for visualization. Before the functional sessions, two resting-state functional connectivity sessions were performed with participants fixating on a cross for the duration of each 6 min session. Images were acquired using a sequence similar to the functional sequence, the only differences being the use of a $2200 \mathrm{~ms}$ TR and the acquisition of 164 volumes of 36 axial slices per session. All BOLD data were processed with SPM5 (Wellcome Department of Imaging Neuroscience). Slice acquisi- 
Table 1. Behavioral measures

\begin{tabular}{lllll}
\hline \multirow{2}{*}{ Measure } & Run & \multicolumn{2}{l}{ Cue validity } & \\
\cline { 3 - 5 } & validity & Valid & Neutral & Invalid \\
\hline Accuracy & High & $0.732(0.110)$ & - & $0.648(0.126)$ \\
& Low & $0.665(0.182)$ & $0.646(0.192)$ & $0.608(0.176)$ \\
Reaction time (s) & High & $1.212(0.204)$ & - & $1.222(0.195)$ \\
& Low & $1.298(0.205)$ & $1.311(0.203)$ & $1.339(0.211)$ \\
\hline
\end{tabular}

Accuracy data correspond to the mean proportion of hits minus the mean proportion of false alarms. Reaction times are to correct responses only. Values in parentheses indicate one SD of the mean.

tion timing correction was performed (by temporally resampling relative to the middle slice collected), followed by rigid body motion correction. Functional volumes were then spatially normalized to a canonical echoplanar template using 12-parameter affine and cosine basis transformations, and resampled to $3 \mathrm{~mm}$ isotropic voxels. Volumes were then spatially smoothed with a $6 \mathrm{~mm}$ Gaussian kernel.

fMRI amplitude analysis. Statistical analyses treated participants as a random effect with volumes treated as a temporally correlated time series and modeled by convolving a canonical hemodynamic response function with a series of delta functions marking the onset of each condition of interest from the presentation of the memory probe. The modeled response duration was $4.5 \mathrm{~s}$. Because of their low frequency, incorrect responses were grouped into a single variable of no interest and not further considered. The parameter estimates of the best-fitting canonical hemodynamic response function for each condition were used in pairwise contrasts and stored as a separate image for each participant. These images were then tested against the null hypothesis of no difference between contrast conditions using one-tailed, repeated measures, $t$ tests. Activations were considered significant and further scrutinized if they consisted of 5 of more contiguous voxels and exceeded an $\alpha$ threshold of 0.001 , a typical threshold for recognition memory research.

Resting and task-evoked functional connectivity ( $f(M R I)$ analysis. Resting-state functional connectivity was examined by extracting time courses from two 8-mm-diameter regions of interest (ROIs; using the MARSBAR toolbox for SPM5) (Brett et al., 2002) and entering these as covariates of interest alongside 18 sources of nonspecific variance (six movement parameters, signal from spheres in the left lateral ventricle, in left hemisphere deep cerebral white matter and averaged across the whole brain, and the nine first derivatives of these covariates) in an SPM general linear model. Resulting maps were subjected to the same threshold as the functional data and illustrate areas whose activation reliably covaries with the seed region(s), on a scan-by-scan basis, after nonspecific effects have been controlled.

Task-evoked connectivity analysis examined the pattern of covariance across ROIs for the mean evoked invalid cueing response of each participant. For this analysis, ROIs were drawn from the contrast map illustrating the effect of invalid versus valid memory cueing. Following this, the mean evoked responses in two key seed regions were correlated with the remaining target ROIs across the 19 participants, yielding one correlation value for each combination of seed region, target region, and experimental contrast of interest. These correlations were then examined to determine whether they reliably differed as a function of experimental factors, such as the run validity (high or low) or the network association of the target region (see Results, Resting and task-evoked connectivity, and Fig. 4 for further details).

\section{Results}

\section{Behavioral data}

Accuracy scores (the mean proportion of hits minus the mean proportion of false alarms) were entered into a cue validity (valid or invalid) by run validity (high or low) repeated-measures ANOVA (neutral cues from low-run-validity sessions were not entered into the ANOVA as there was no equivalent condition in high-run-validity sessions) (see Table 1 ). There were main effects of cue validity, $F_{(1,18)}=7.485, p<0.05$, and run validity, $F_{(1,18)}=$ $4.432, p<0.05$, and no significant interaction, $F<1$. As would be expected if participants were incorporating the cues into their memory judgments, across all sessions valid cues yielded greater accuracy than invalid cues, and this translated into higheraccuracy scores for the high-run-validity sessions, which contained a greater proportion of valid cues than the low-run-validity sessions.

Mean reaction times to correct responses, collapsed across item status, were also entered into an equivalent ANOVA (see Table 1). There was a main effect of run validity, $F_{(1,18)}=15.44$, $p<0.005$, but no main effect of cue validity, $F_{(1,18)}=1.55$, n.s., and no interaction, $F<1$. Participants responded faster to items in high- versus low-run-validity sessions, but across all sessions there was no effect of cue validity on reaction time.

\section{fMRI data}

Active manipulation of mnemonic expectancy

To generally assess the effects of cue validity (valid vs invalid) and item status (hits vs correct rejections), the data were collapsed across all sessions (high and low run validity). Consistent with prior research, the standard retrieval success contrast of hits versus correct rejections yielded significant activation in areas including left lateral premotor cortex $(\sim \mathrm{BA} 8)$ and bilateral dorsal parietal regions $(\sim \mathrm{BA} 7 / 40)$ extending from the SMG dorsally to the superior parietal lobule (SPL) (Table 2, Fig. 2A). Bilateral posterior middle temporal gyrus regions (pMTG; BA 21) and bilateral caudate regions were also implicated. The reverse contrast, correct rejections versus hits, yielded no significant activation.

In contrast to the modest lateral parietal activation differences observed under the item contrast, the orthogonal invalid cueing effect (invalid cueing vs valid cueing) contrast revealed prominent and extensive effects (Fig. $2 \mathrm{~B}$ ) [a valid cueing versus invalid cueing contrast was also performed but is not reported due to minimal activation (one 5-voxel suprathreshold activation in left hemisphere white matter and one 16-voxel suprathreshold activation in right hemisphere white matter)]. Collapsed across whether items were studied or novel, when the recognition evidence conflicted with cued expectations, activation was seen in a number of regions, including medial PFC $(\sim \mathrm{BA} 8 / 9 / 10)$, bilateral dorsal premotor $(\sim \mathrm{BA} 6 / 9)$ and frontopolar $\mathrm{PFC}(\sim \mathrm{BA}$ $10)$, bilateral insula extending to inferior frontal gyrus, bilateral caudate, bilateral inferior parietal lobule (IPL; BA 39/40) incorporating supramarginal gyrus (SMG) and AG and extending ventrally through to middle temporal gyrus (MTG; BA 21/ 22 ) and right hippocampus (Table 3, Fig. $2 B$ ). In the left hemisphere, the lateral parietal region demonstrating a retrieval success effect largely fell within or superior to the parietal region sensitive to cue validity. This activation region was likely not independent of cueing effects. Indeed, when the success contrast was repeated after exclusively masking all regions demonstrating invalid cueing effects at a $p<0.005$ threshold (so that only voxels with a specificity for retrieval success would survive the masking procedure), the lateral parietal response was virtually eliminated, and it was completely eliminated when the masking threshold was further lowered to 0.05 . Thus overall, there were no lateral parietal responses in the current study that reflect successful retrieval and that are simultaneously insensitive to expectancy violations. In contrast, the vast majority of the lateral parietal response was governed by the correspondence between the anticipatory cue and the memory probe status (cf. Vilberg and Rugg, $2009 \mathrm{~b}$ ). This suggests that the interpretation of the parietal response, particularly that of the IPL, in terms of successful episodic retrieval is inappropriate and that the response is instead linked to expectancy violation and perhaps the subsequent exertion of 
Table 2. Regions demonstrating significant increases in response to hits versus correct rejections

\begin{tabular}{|c|c|c|c|c|c|c|c|}
\hline Region & Lat. & $\mathrm{BA}$ & $x$ & $y$ & $z$ & Vox. & Zscore \\
\hline \multicolumn{8}{|l|}{ Parietal } \\
\hline SPL/SMG & $\mathrm{L}$ & $7 / 40$ & -36 & -60 & 60 & 327 & 4.79 \\
\hline$A G$ & $\mathrm{~L}$ & 39 & -51 & -66 & 39 & 12 & 3.59 \\
\hline Precuneus & $\mathrm{L}$ & 7 & -15 & -63 & 69 & 5 & 3.34 \\
\hline \multirow[t]{2}{*}{$\mathrm{SPL}$} & $\mathrm{R}$ & 7 & 36 & -72 & 51 & 58 & 4.22 \\
\hline & & 7 & 12 & -66 & 66 & 11 & 3.90 \\
\hline Postcentral gyrus & $\mathrm{R}$ & 2 & 48 & -30 & 54 & 14 & 3.74 \\
\hline SMG & $\mathrm{R}$ & 40 & 45 & -42 & 63 & 16 & 3.40 \\
\hline \multicolumn{8}{|l|}{ Cingulate } \\
\hline Posterior cingulate & $\mathrm{L} / \mathrm{R}$ & - & -12 & -42 & 27 & 92 & 4.35 \\
\hline \multicolumn{8}{|l|}{ Temporal } \\
\hline \multirow[t]{2}{*}{ MTG } & $\mathrm{L}$ & 21 & -63 & -48 & -6 & 21 & 4.26 \\
\hline & & 21 & -66 & -30 & -3 & 5 & 3.64 \\
\hline White matter & $\mathrm{L}$ & - & -45 & -24 & -12 & 7 & 3.65 \\
\hline MTG & $\mathrm{R}$ & 21 & 57 & -42 & -9 & 33 & 4.30 \\
\hline White matter & $\mathrm{R}$ & - & 30 & -66 & 12 & 6 & 4.03 \\
\hline \multicolumn{8}{|l|}{ PFC } \\
\hline Premotor & $\mathrm{L}$ & 8 & -36 & 15 & 51 & 69 & 3.99 \\
\hline MFG & $\mathrm{L}$ & 9 & -42 & 27 & 36 & 5 & 3.77 \\
\hline \multicolumn{8}{|l|}{ Cerebellum } \\
\hline Anterior lobe & $\mathrm{L}$ & - & -24 & -51 & -30 & 6 & 3.78 \\
\hline Posterior lobe & $\mathrm{L}$ & - & -36 & -60 & -30 & 6 & 3.75 \\
\hline \multicolumn{8}{|l|}{ Brainstem } \\
\hline Midbrain & $\mathrm{L}$ & - & -6 & -21 & -18 & 6 & 3.78 \\
\hline Midbrain & $\mathrm{R}$ & - & 15 & -24 & -15 & 8 & 3.81 \\
\hline \multicolumn{8}{|l|}{ Basal ganglia } \\
\hline Caudate & $\mathrm{L}$ & - & -9 & 12 & 0 & 18 & 3.75 \\
\hline \multirow[t]{2}{*}{ Caudate } & $\mathrm{R}$ & - & 12 & 12 & 12 & 8 & 3.83 \\
\hline & & - & 9 & 15 & -3 & 23 & 3.72 \\
\hline \multicolumn{8}{|l|}{ Thalamus } \\
\hline Anterior thalamus & $\mathrm{L} / \mathrm{R}$ & - & 0 & -3 & 9 & 41 & 2.96 \\
\hline Posterior thalamus & $\mathrm{R}$ & - & 0 & -33 & 6 & 25 & 3.75 \\
\hline
\end{tabular}

Listed regions are SPM clusters containing at least 5 significant voxels. $x, y$, and $z$ coordinates refer to cluster maxima. Lat., Laterality; BA, approximate Brodmann's location; Vox., number of significant voxels; SPL, superior parietal lobule; SMG, supramarginal gyrus; AG, angular gyrus; PFC, prefrontal cortex; MTG, middle temporal gyrus; MFG, middle frontal gyrus. Coordinates are in MNI space.

cognitive control required to render the correct, noncued decisions (Christoff and Gabrieli, 2000; Braver and Bongiolatti, 2002; Dobbins et al., 2002; Bunge et al., 2005).

Because the invalid cueing effect contrast is orthogonal to item status (hits vs correct rejections) and run validity (high vs low), it was used to define unbiased ROIs to see how these two factors may have systematically influenced the left parietal response. Two left parietal regions (Fig. 2Ci: SMG and AG) were considered based on their dissociation in the resting-state connectivity and functional connectivity analyses below (see Resting and task-evoked connectivity and Fig. 2 for cluster definitions). Additionally, similar regions have been shown to dissociate based on the quality of subjective memory reports in prior fMRI studies (Henson et al., 1999; Yonelinas et al., 2005; Daselaar et al., 2006). In both parietal regions, there was an elevated response to invalid cueing trials compared to valid cueing trials, and this increase did not interact with run validity (high or low) or the status of the item (hit or correct rejection) (Fig. 2Cii). Critically, the mean $\beta \mathrm{s}$ demonstrate the greater sensitivity of these regions to cue validity than item status. Although there was a main effect of item status, with hits demonstrating greater activation than correct rejections, $F_{(1,18)}=10.971, p<0.01$, the main effect of cue validity was much larger, $F_{(1,18)}=24.933, p<0.001$. Numerically, the average difference between invalid and valid cueing $\beta$ s was more than threefold greater than the average difference between hit and correct rejection $\beta$ s.

Overall, the analyses demonstrate that the invalid cueing response occurs for both new and studied items. Since new items are likely to evoke less episodic content than old items (Mather et al., 1997), the network's functional response cannot be strictly dependent upon successful episodic retrieval since the spurious retrieval of episodic information for new items would tend to lead to incorrect, not correct, responding (cf. Lyle and Johnson, 2007).

\section{Resting and task-evoked connectivity}

Vincent et al. (2008) used resting connectivity analysis to identify a putative frontoparietal control network that is partially overlapping with regions identified in the current invalid cueing effect map. As noted by these researchers, functional interpretations based on resting-state data and reverse inference in isolation are risky (Poldrack, 2006), and a direct manipulation of cognitive control demands is needed to confirm that this network is heavily linked with cognitive control. The current findings provide this more direct test; however, we sought to more firmly determine the correspondence between the task-evoked invalid cueing response and the pattern of resting connectivity within the same group of participants. Based on prior work examining cognitive control and episodic retrieval (Botvinick et al., 2001; Kouneiher et al., 2009), both of which are presumably required to render correct responses despite invalid cueing, two 8-mm-diameter seeds along the medial prefrontal cortex were used to investigate resting connectivity: a dorsomedial PFC seed and an anteromedial PFC seed (see Fig. 3A; for further seed choice rationale, see supplemental material S1, available at www.jneurosci.org). The dorsomedial seed (center $[3,24,51]$ in MNI space) yielded a resting-state network displaying considerable spatial overlap 
with the task-evoked response during invalid cueing and closely corresponds to the frontoparietal control network identified in Vincent et al. (2008). This network includes a large region of PFC activation including the dorsomedial region $(\sim \mathrm{BA} 8 / 9)$, bilateral frontopolar cortex ( $\sim$ BA 10/11), dorsal premotor cortex $(\sim \mathrm{BA} 6 / 8)$, and inferior frontal gyrus $(\sim$ BA $44 / 45)$, as well as bilateral caudate, bilateral IPL ( $\sim$ BA 40$)$, and bilateral inferior temporal gyrus $(\sim \mathrm{BA} 37)$ (Fig. 3B).

The anteromedial seed (center $[0,63$, 27] in MNI space) recovered other regions of the task-evoked map that were not recovered by the dorsomedial seed, including the inferotemporal areas $(\sim \mathrm{BA}$ $20 / 21)$, superior frontal gyrus areas $(\sim B A$ $6 / 8 / 9$ ), and bilateral hippocampus/amygdala (Fig. 3B). Critically, these two relatively proximal seed regions implicated largely disjoint networks despite the fact that the separate and independent connectivity analyses did not demand this outcome. Of the 13,523 voxels implicated across the two connectivity maps, only $7.9 \%$ were common to both. Additionally, when both seed regions were simultaneously present in the same model and directly contrasted, the disjoint network pattern was preserved demonstrating it was not simply a thresholding artifact but a statistically reliable difference in interconnectivity patterns (Fig. 3C). Overall, the resting-state analyses demonstrate two dissociable networks that largely recover all of the regions implicated in the invalid cueing, task-evoked response, with the exception of occipital extrastriate regions. This spatial convergence is also apparent in conjunction map of the invalid cueing effect contrast and the two restingstate networks (Fig. 3D). Given that resting connectivity and task-evoked maps were each thresholded at $p<0.001$, this demonstrates a compelling correspondence between the task-evoked response to invalid versus valid cueing, and the pattern of interregional covariance observed at rest in the same participants (Fig. $2 B$ ).

Critically, in terms of the current parietal focus, the resting connectivity analysis demonstrated functional heterogeneity in the left IPL. Whereas the SMG area was preferentially connected with the dorsomedial seed, the AG area was preferentially connected with the anteromedial seed. This finding demonstrates that although the regions do not dissociate based on the amplitude of the taskevoked response (see Active manipulation of mnemonic expectancy), they do dissociate based on their connectivity at rest. Based on this later finding, we examined whether the connectivity patterns during the active task also demonstrated a functional dissociation of left SMG and AG regions. To examine this, the SMG and AG clusters in Figure 2Ci were used in a task-evoked functional connectivity
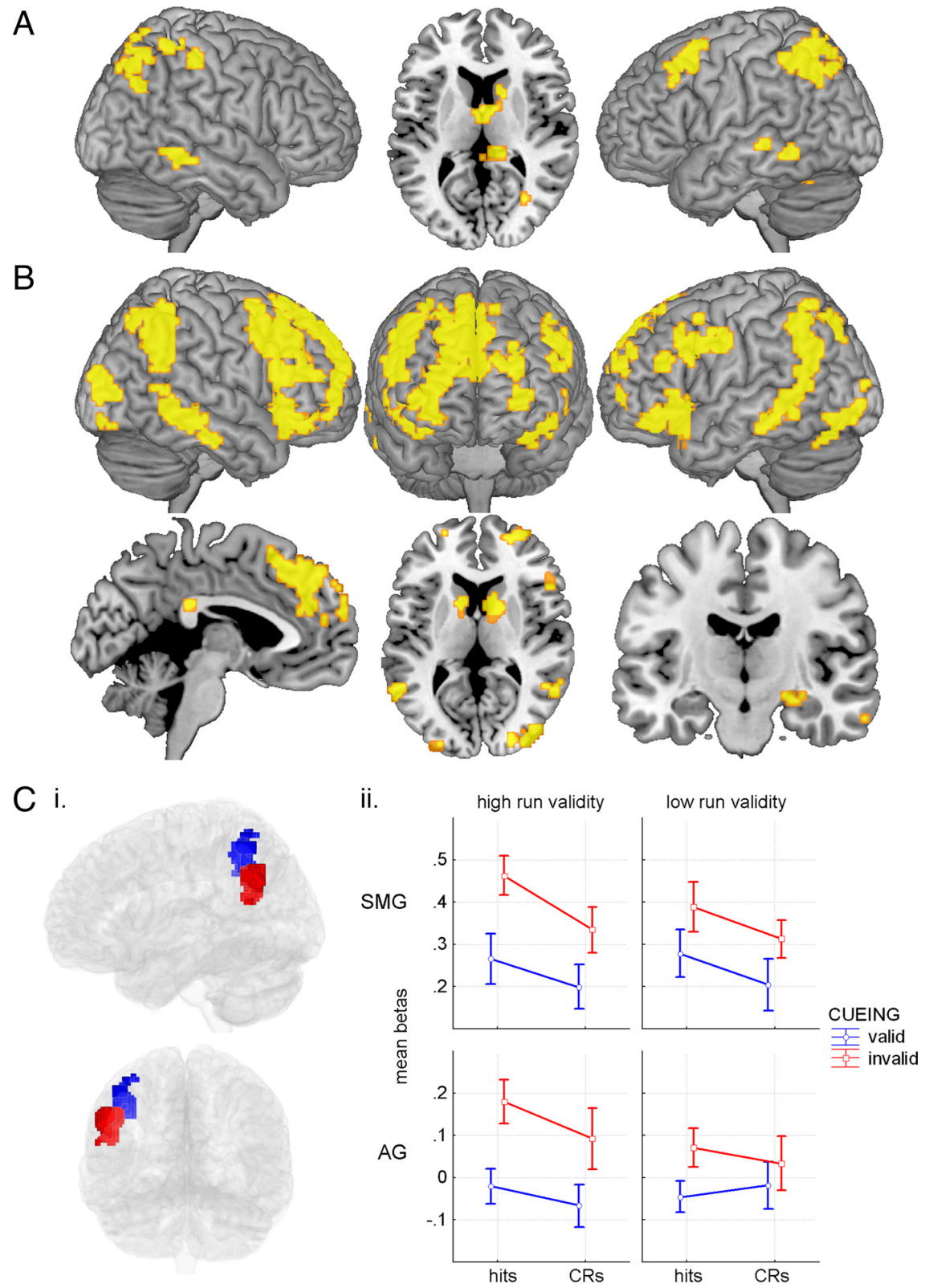

Figure 2. Task-evoked maps and amplitude responses. $\boldsymbol{A}$, Regions demonstrating significant activation in the retrieval success contrast (hits vs correct rejections). $\boldsymbol{B}$, Regions demonstrating significant activation in the invalid cueing contrast (invalid cueing vs valid cueing). Ci, The 256 voxel SMG (blue) cluster was defined by inclusively masking the left hemisphere temporoparietal cluster from the invalid cueing contrast map (thresholded at $p<0.001,5$ contiguous voxels) with the dorsomedial seed resting-state map, exclusive of voxels also lying in the anteromedial seed map (masks thresholded at $p<0.001$ ) (for further explanation of resting-state seeds and maps, see Results, Resting and task-evoked connectivity, and Fig. $3 A, B$ ). The 314 voxel AG (red) cluster was defined in a similar way, by inclusively masking the parietal portion of the left hemisphere, invalid cueing, temporoparietal cluster with the anteromedial seed resting-state map exclusive of voxels in the dorsomedial seed map. Clusters are viewed from left and posterior aspects. Cii, Mean $\beta$ parameter estimates from SMG (top panels) and AG clusters (bottom panels) in response to valid and invalid cueing. Error bars represent SEs. CRs, Correct rejections.

analysis. The mean invalid cueing effect (invalid minus valid cueing $\beta s$ ) in the two parietal clusters (SMG and AG) was cross-correlated with the remaining 64 target ROIs implicated in the SPM, across the 19 participants, for each combination of run validity (high or low) and item status (hit or correct rejection). See Figure 4, $A$ and $B$, for a summary of the analysis. If the pattern of connectivity observed at rest constrains the pattern of connectivity in task-evoked responses, then the SMG and AG regions should again dissociate in the degree to which they correlate with target ROIs coincident with the two different networks identified in the resting connectivity analysis. 
Table 3. Regions demonstrating significant increases in correct response to invalid cues versus valid cues

\begin{tabular}{|c|c|c|c|c|c|c|c|}
\hline Region & Lat. & $\mathrm{BA}$ & $x$ & $y$ & $Z$ & Vox. & Zscore \\
\hline \multicolumn{8}{|l|}{ PFC } \\
\hline IFG/insula & $\mathrm{L}$ & 47 & -27 & 18 & -9 & 344 & 5.94 \\
\hline Frontopolar & $\mathrm{L}$ & 10 & -30 & 60 & 3 & 21 & 4.87 \\
\hline Dorsal premotor & $\mathrm{L}$ & $6 / 44$ & -39 & 6 & 36 & 166 & 4.01 \\
\hline SFG & $\mathrm{L}$ & 8 & -12 & 36 & 60 & 6 & 3.52 \\
\hline \multirow[t]{2}{*}{ MFG } & $\mathrm{L}$ & 46 & -48 & 36 & 24 & 22 & 3.43 \\
\hline & & 6 & -45 & 12 & 54 & 6 & 3.37 \\
\hline Frontopolar/dorsal medial//FG/insula & $\mathrm{L} / \mathrm{R}$ & $6 / 8 / 9 / 10 / 47$ & 27 & 60 & 18 & 1555 & 4.67 \\
\hline \multicolumn{8}{|l|}{ Occipital } \\
\hline Inferior occipital gyrus & $\mathrm{L}$ & 19 & -42 & -84 & -9 & 89 & 4.64 \\
\hline Inferior occipital gyrus & $\mathrm{R}$ & 19 & 39 & -87 & -12 & 9 & 3.66 \\
\hline Middle occipital gyrus & $\mathrm{L}$ & 19 & -30 & -96 & 12 & 7 & 3.83 \\
\hline Middle occipital gyrus & $\mathrm{R}$ & $18 / 19$ & 42 & -81 & 6 & 104 & 4.38 \\
\hline \multicolumn{8}{|l|}{ Parietal/temporal } \\
\hline SMG/AG/STG/MTG & $\mathrm{L}$ & $21 / 22 / 39 / 40$ & -42 & -54 & 42 & 335 & 4.50 \\
\hline Precuneus/SPL & $\mathrm{L}$ & 7 & -21 & -78 & 42 & 53 & 3.78 \\
\hline SMG & $\mathrm{R}$ & 40 & 42 & -54 & 39 & 401 & 5.02 \\
\hline \multirow[t]{2}{*}{ MTG } & $\mathrm{R}$ & 21 & 63 & -45 & -6 & 152 & 4.55 \\
\hline & & 21 & 51 & -51 & 6 & 10 & 3.28 \\
\hline Hippocampus & $\mathrm{R}$ & - & 21 & -15 & -12 & 9 & 3.66 \\
\hline \multicolumn{8}{|l|}{ Basal ganglia } \\
\hline Caudate & $\mathrm{L}$ & - & -12 & -3 & 18 & 30 & 3.63 \\
\hline Caudate & $\mathrm{R}$ & - & 15 & 6 & -3 & 92 & 4.70 \\
\hline \multicolumn{8}{|l|}{ Cingulate } \\
\hline Posterior cingulate & $\mathrm{L}$ & 23 & -6 & -30 & 27 & 6 & 3.74 \\
\hline Anterior cingulate & $\mathrm{R}$ & 32 & 12 & 33 & 24 & 5 & 3.37 \\
\hline
\end{tabular}

Listed regions are SPM clusters containing at least 5 significant voxels. $X$, $y$, and $z$ coordinates refer to cluster maxima. Lat., Laterality; BA, approximate Brodmann's location; Vox., number of significant voxels; $\mathrm{PFC}$, prefrontal cortex; IFG, inferior frontal gyrus; $\mathrm{SFG}$, superior frontal gyrus; $\mathrm{MFG}$, middle frontal gyrus; $\mathrm{SMG}$, supramarginal gyrus; $\mathrm{AG}$, angular gyrus; $\mathrm{STG}$, superior temporal gyrus; $\mathrm{MTG}$, middle temporal gyrus; $\mathrm{SPL}$, superior parietal lobule. Coordinates are in MNI space.

That is, the SMG region should preferentially correlate with ROIs falling in the dorsomedial seed network and the AG should preferentially correlate with ROIs falling in the anteromedial seed network. Additionally, we tested whether any patterns of connectivity were influenced by the active task characteristics, namely, the run validity and the study status of the probes.

Figure $4 C$ illustrates the connectivity findings via a mixed ANOVA with factors of run validity (high or low), item status (hit or correct rejection), seed region (SMG or AG), and target network (dorsomedial or anteromedial). The last factor was a grouping factor in which each target ROI was classified as falling within the dorsomedial seed network or the anteromedial seed network. ROIs falling outside of the two networks, or falling within areas of network overlap were not considered. The dependent variable was the crosscorrelation value between the seed and target regions across the 19 participants. The ANOVA yielded a significant four-way interaction across the factors, $F_{(1,62)}=40.920, p<0.001$. Follow-up tests were conducted examining the key combinations of the four factors (Fig. $4 C i-i v)$. Under conditions of high run validity, clear evidence of network selectivity was obtained. For hit trials (Fig. 4Ci), the SMG and $\mathrm{AG}$ regions demonstrated a cross-over dissociation, $F_{(1,62)}=$ 36.873, $p<0.001$, in the correlation values. The SMG correlations were significantly higher for target ROIs in the dorsomedial seed network than those in the anteromedial seed network, $t_{(62)}=3.052$, $p<0.01$. The reverse pattern held for the AG correlations, which instead were significantly higher for ROIs in the anteromedial seed network than those in the dorsomedial seed network, $t_{(62)}=5.784$, $p<0.001$. This pattern corresponds with that of the resting-state data and further supports the functional segregation of the two networks and of the SMG and AG parietal regions in particular. Although the average correlation between the seed regions and the target network ROIs remained high in the remaining cells of the design (Fig. 4Cii-iv), the high degree of network selectivity in the response of the seed regions was clearly absent. Differences in the mean levels of correlation (i.e., main effects of the factors) are not interpreted because these may simply reflect general arousal effects that are unrelated to questions of functional heterogeneity of the left inferior parietal region. Overall, the task-evoked functional connectivity analysis demonstrated that SMG and AG differ in the degree to which they couple with ROIs in the two independently defined resting connectivity networks. This selective coupling appears restricted to conditions under which the cues evoke high expectation that the upcoming items are new, but positive retrieval evidence countermands this expectation (Fig. 4Ci). However, this network selectivity is not fixed, as the remaining cells of the design did not demonstrate this clear network selectivity in the response of the SMG and AG regions. Thus the network selectivity of the SMG and AG evoked response is most prominent when unexpectedly old items are encountered. As with the resting-state connectivity analysis, these findings demonstrate that analyzing the data solely in terms of the amplitude of BOLD response may miss functional dissociations clearly present in the patterns of connectivity across the cortex.

\section{Summary of $\mathrm{AMRI}$ findings}

The fMRI data demonstrate that the left lateral parietal recognition response is highly sensitive to mnemonic expectancy violation and that SMG and AG regions are functionally dissociable based on their differential functional connectivity. In terms of amplitude data, the left lateral parietal region demonstrated invalid cueing effects that occurred for old and new items. This invalid cueing effect was far more robust than the small retrieval success effect present in the data. Although the amplitude data suggested no functional difference between SMG and AG regions, the connectivity data demonstrated that the two regions are preferentially coupled with dorsomedial and anteromedial PFC seed networks. This coupling is present during 
A

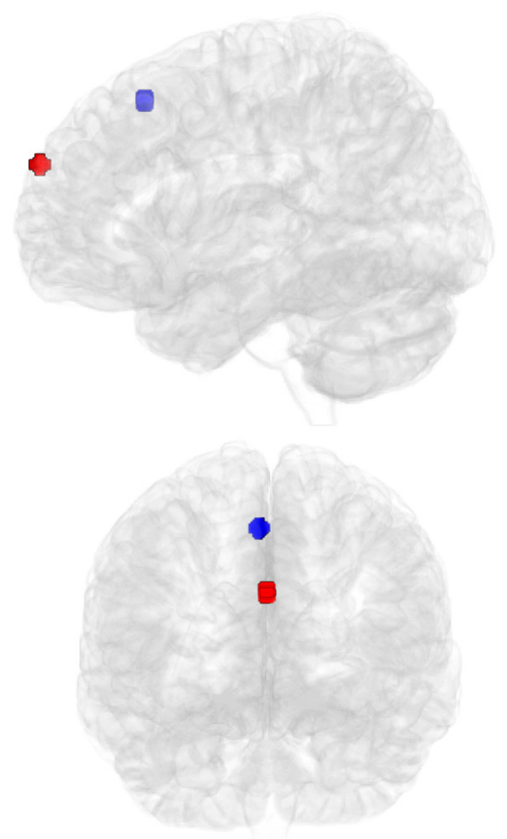

C

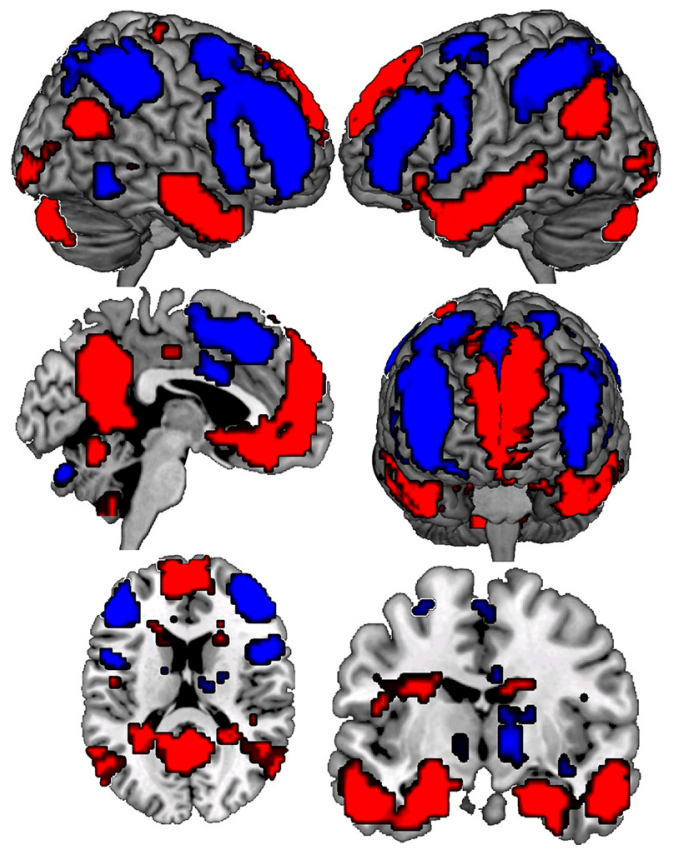

B
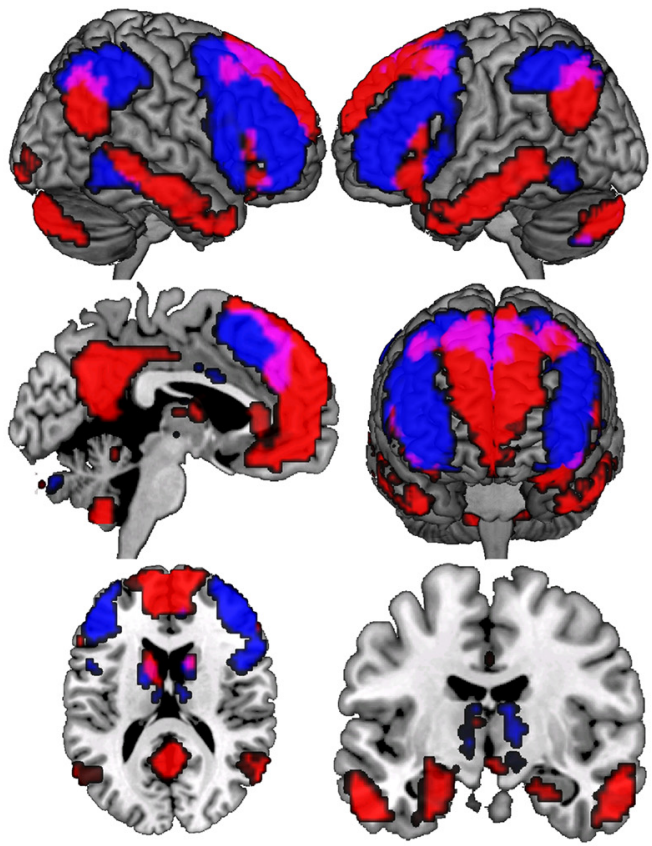

$\mathrm{D}$
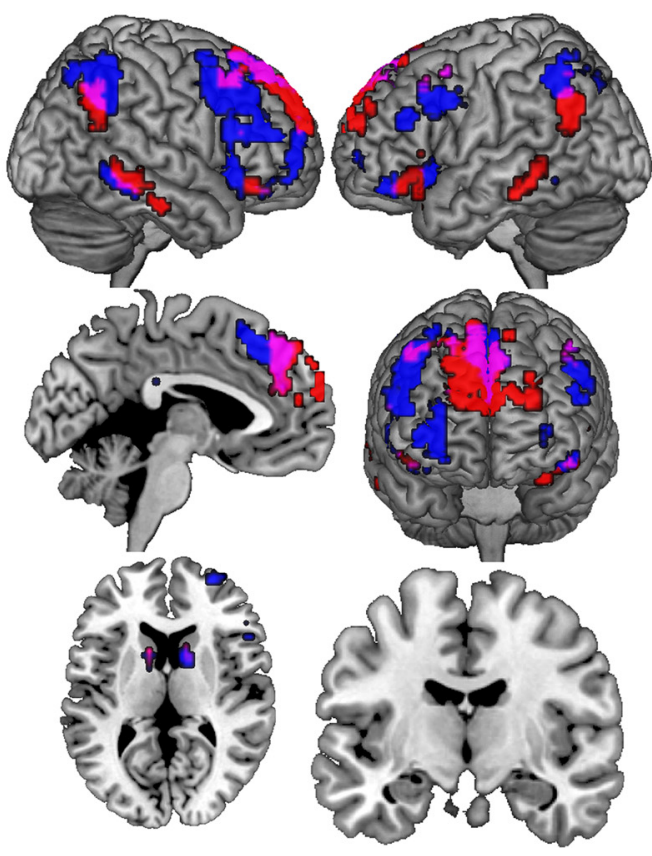

Figure 3. Resting-state seeds and maps. $\boldsymbol{A}$, ROls used to seed resting-state analyses: dorsomedial PFC ROI (an 8-mm-diameter sphere with center [3, 24, 51]; blue); anteromedial PFC ROI (an 8-mm-diameter sphere with center [0,63, 27]; red). ROls are viewed from left and anterior aspects. $\boldsymbol{B}$, Resting-state network of regions demonstrating significant activation associated with the activation of the dorsomedial seed (blue) and the activation of the anteromedial seed (red; overlap in purple; thresholded at $p<0.001,5$ contiguous voxels). $\boldsymbol{C}$, Maps of the same seed regions (dorsomedial seed network, blue; anteromedial seed network, red) entered into one model and directly contrasted (thresholded at $p<0.001,5$ contiguous voxels). D, (onjunction map of regions demonstrating activation associated with the conjunction of the invalid cueing effect contrast (thresholded at $p<0.001,5$ contiguous voxels) and anteromedial seed resting-state network (thresholded at $p<0.001,5$ contiguous voxels; red) and dorsomedial seed resting-state network (thresholded at $p<0.001,5$ contiguous voxels; blue; overlap in purple).

passive resting scans and present during the active task, when unexpectedly old items are encountered. These findings are unanticipated from a strictly retrieval-based interpretation of the lateral parietal response.

\section{Subject differences in parietal recruitment}

The above analyses demonstrate that dissociable SMG and AG parietal regions respond when expectations regarding subsequent mnemonic experiences are violated. However, the design is considerably different from standard recognition studies implicating the left lateral parietal region. Given this, we reanalyzed a portion of an extant recognition dataset (S. Han, S. A. Huettel, A. Raposo, R. A. Adcock, and I. G. Dobbins, unpublished observations) to see whether the pattern of individual variation in activation also favored an expectancy violation interpretation over a retrieval success interpretation (for design details, see supplemental material S2, available at www.jneurosci.org). The analysis focused on the initial two scans of the experiment, which examined standard verbal 


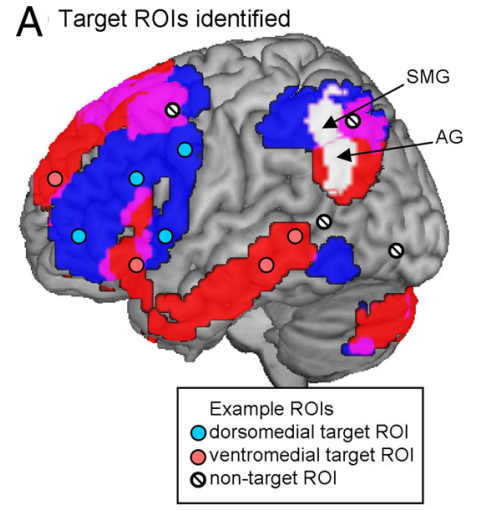

B Differential beta correlations calculated for each ROI

C

Correlation coefficients entered into ANOVA hits

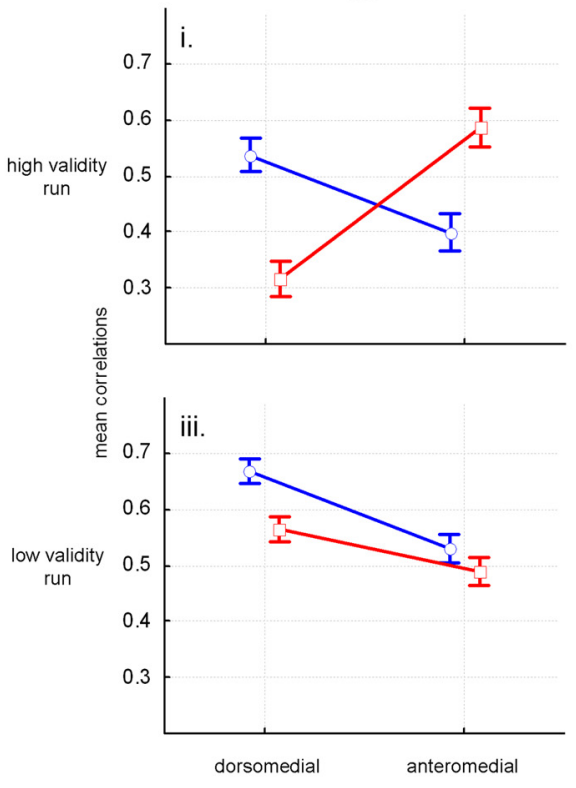

e.g.

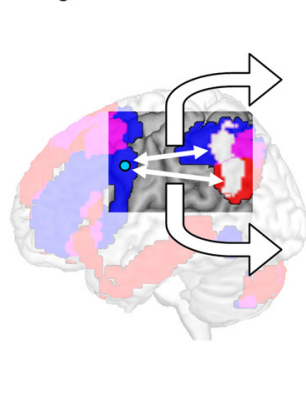

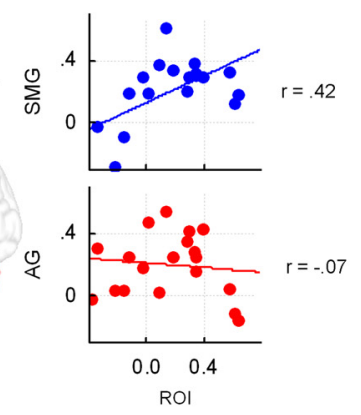

CRs ii.

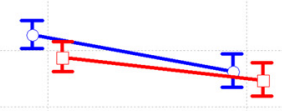

iv.
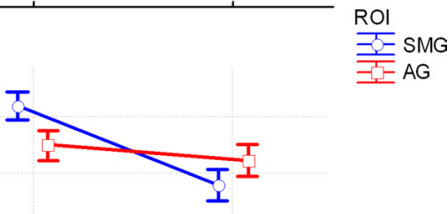

target ROI network membership

Figure 4. Schematic diagram and results of task-evoked connectivity analysis. These analyses demonstrating the degree to which AG and SMG seeds differ in their coupling with ROIs in the two independently defined resting connectivity networks. $A$, ROIs were 115 8-mm-diameter spheres centered on local maxima from the invalid cueing contrast (not shown in this figure; see Fig. 2B). ROls were identified as target ROIs if their centers lay within either the dorsomedial seed (35) or anteromedial seed (29) resting-state maps (blue and red, respectively). ROls with centers lying in both resting-state maps (9) or neither map (42) were excluded from this analysis. A small number of spheres representing possible target and nontarget ROIs are shown for illustrative purposes. SMG and AG clusters are shown in white (see Fig. 2 Ci for details of how they were defined). $\boldsymbol{B}$, Differential $\beta$ s (invalid minus valid cueing $\beta$ s) from all target ROIs were cross-correlated (across all 19 participants) with differential $\beta$ s from the SMG and AG clusters (see Fig. $2 A$ ), producing two correlation coefficients per target ROI for each combination of run validity (high or low) and item status (hit or correct rejection). Correlations between differential $\beta$ s from one target ROI and SMG/AG clusters, under one run-status combination (hits under high run validity), are shown. C, Correlation coefficients were entered into an ANOVA. Means are broken down in the four panels (i-iv) by run validity (rows) and item status (hits, correct rejections; columns) with each panel showing the mean correlation coefficients between $\beta$ s (invalid minus valid cue trials) from SMG (blue) and AG clusters (red) and dorsomedial (left) and anteromedial target ROls (right). Error bars represent SEs. CRs, Correct rejections.

recognition in an event-related design with neither feedback nor precueing. If the contrast of hits versus correct rejections is closely linked to the successful recovery of episodic content, then highly accurate observers should evidence a greater differential activation than less accurate observers. In contrast, if the response is linked to the violation of memory expectations, then it should not depend strictly on accuracy but may instead be linked to individual differences in response biases.

Separate simple regressions of accuracy $\left(d^{\prime}\right)$ and response bias (c) onto the contrast of hits versus correct rejections support the expectancy violation interpretation. There were no regions sen- sitive to the individual variation in accuracy. In contrast, a reliable correlation with response bias occurred in parietal and other regions overlapping with those demonstrating an invalid cueing effect in the current data (Fig. 5A). More specifically, individuals with a conservative response bias demonstrated enhanced differential activation for hits versus correct rejections compared to more lax individuals, yet this same differential response was unrelated to accuracy differences among the individuals. The convergence with the invalid cueing effect maps is illustrated in the conjunction map in Figure $5 B$ and demonstrates strong regional correspondence between these two analyses of different datasets. To further illustrate this correspondence, an $8 \mathrm{~mm}$ sphere from a lateral parietal local maximum identified by the invalid cueing contrast was used as an unbiased ROI from which differential $\beta$ s (hits minus correct rejections) were extracted from this standard recognition dataset. These $\beta$ s from each participant were correlated with response bias, $r=0.624, p<0.005$, but not accuracy, $r=-0.096$, n.s., demonstrating that lateral regions associated with an elevated invalid cueing response, also show a reliable dependence between individual differences in response bias and the relative activation elicited by hits versus correct rejections. We discuss the significance of this novel finding further in the discussion.

\section{Discussion}

The "retrieval success" contrast yields a very reliable network of activation in the functional imaging literature (e.g., Konishi et al., 2000; McDermott et al., 2000; Wagner et al., 2005; Vilberg and Rugg, 2008), implicating both superior and inferior parietal regions. Responses in the vicinity of the IPS and SMG have been characterized in terms of top-down, directed attention toward recovered episodic content (Cabeza et al., 2008), a reflection of the relative familiarity of stimulus materials (Vilberg and Rugg, 2008) or as an episodic output buffer (Wagner et al., 2005). More ventral and posterior IPL activations in the vicinity of the AG have also been interpreted as recollection-linked orienting responses (Cabeza et al., 2008) or markers of recollective recovery (Vilberg and Rugg, 2009a,b). Many of these interpretations share two characteristics. First, they interpret responses as closely linked to successful episodic retrieval. For example, the direction of attention toward episodic content, the accumulation of such content in a buffer, or the capture of attention by such content all require that some episodic information be successfully recovered. Second, they do not heavily emphasize the role of observer expectations in governing the responses (cf. Herron et 
A
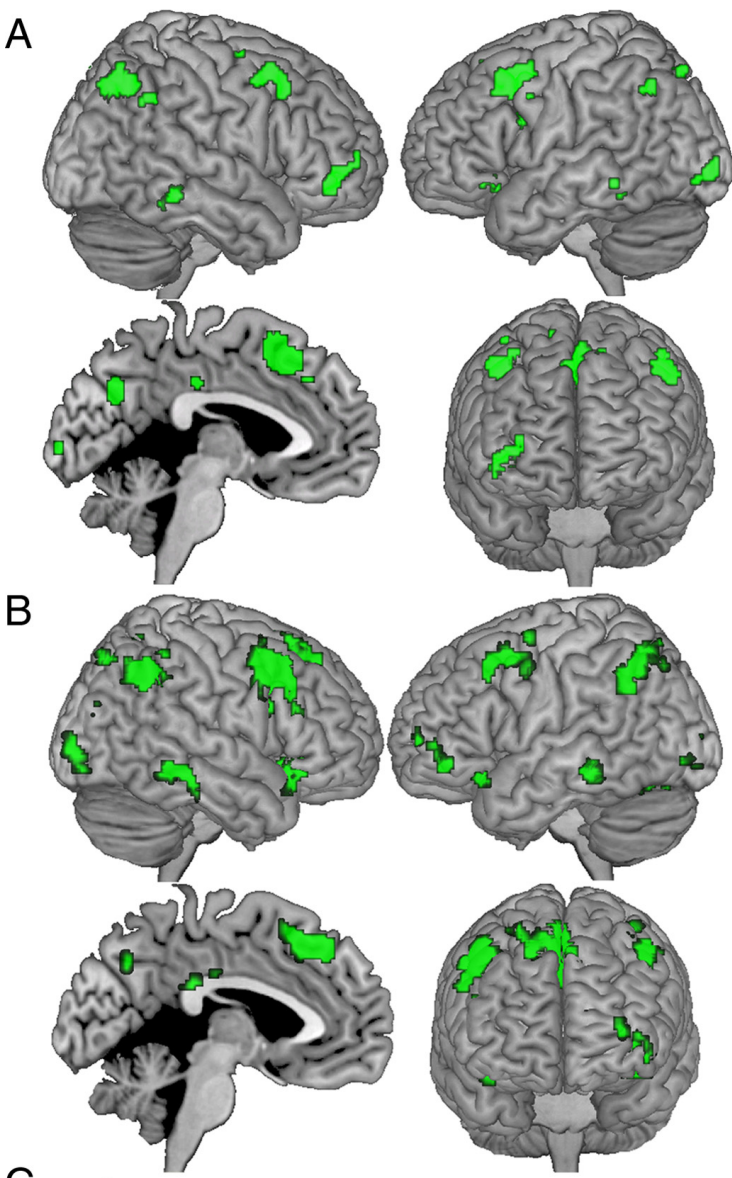

C
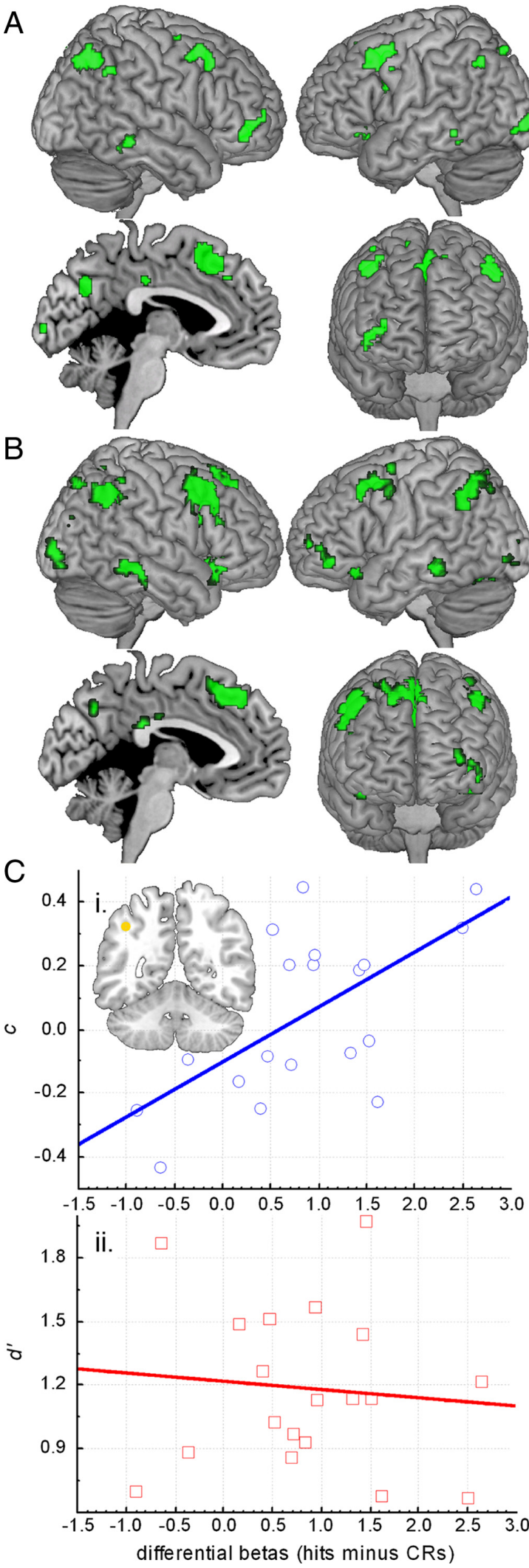

Figure 5. Response bias correlation maps. $\boldsymbol{A}$, Regions demonstrating significant correlation with response bias. $\boldsymbol{B}$, Multiplicative conjunction map of regions demonstrating activation associated with the conjunction of response bias correlation and invalid cueing effect maps (both thresholded at $p<0.005,5$ contiguous voxels). C, Scatter plots showing differential $\beta$ s (hits minus correct rejections) from an independently defined ROl in lateral parietal cortex (an 8-mm-diameter sphere with center $[-42,-54,42]$; shown in yellow on coronal section) plotted against the following: response bias $(c)(i)$ and accuracy $\left(d^{\prime}\right)(i i)$. al., 2004; Vilberg and Rugg, 2009b). The current data are thus important because they demonstrate that both superior and inferior aspects of the lateral parietal response are not strictly dependent upon episodic retrieval but are primarily governed by the match between expectations and subsequent retrieval experiences.

In the current cueing paradigm, there were no lateral parietal regions demonstrating retrieval success responses that were also insensitive to expectancy violations. Additionally, the SMG invalid cueing amplitude response occurred even for correctly identified new items and thus episodic recovery is not a necessary component of this response. Further, this region was shown to track individual differences in decision bias, not retrieval accuracy, during simple uncued recognition. This latter finding is also incompatible with retrieval-based accounts and its link to an expectancy violation interpretation becomes clearer when one considers that more conservative individuals subjectively experience far fewer items as "old." For illustration, consider two observers with the same level of discrimination $\left(d^{\prime}=1.0\right)$ but considerably different decision biases [strict $(c=1.0)$ and neutral $(c=0.0)$ ]. Under these circumstances, the strict observer is half as likely as the neutral observer to identify any given item as "old" ( 0.33 vs 0.67 "old" response rates). Within the expectancy violation framework, this means the strict observer must countermand a general expectation that items are new to successfully execute a correct "old" decision. The more conservative the individual, the greater the need to overcome this general expectation-hence the correlation between decision bias and parietal activation in Figure 5. Similar findings exist in oddball detection research, where decreasing the frequency of targets increases the response to them in the SMG region (McCarthy et al., 1997; Linden et al., 1999; Stevens et al., 2000; Horovitz et al., 2002). The criterion findings in the current study are consistent with this general operating principle and the first to demonstrate that individual differences in subjective biases differentially affect the recognition response independent of accuracy. The final piece of evidence in the current data linking the SMG region to expectancy violation, and not episodic retrieval, is its colocalization in the dorsomedial seed resting connectivity network (Fig. 3). This network was seeded with an anterior cingulate/pre-SMA cortex region. Prior literature firmly implicates anterior cingulate in general cognitive control (Botvinick et al., 2001; Kouneiher et al., 2009), which in the current procedure is critical for countermanding the anticipatory cues when memory evidence mismatches the observers expectations. Additionally, unlike the anteromedial seed network, the dorsomedial seed network did not implicate lateral and medial temporal lobe regions that support semantic and episodic memory retrieval, respectively. Thus when considered in total, the current study provides multiple lines of evidence linking the SMG response to the violation of memory expectation and the associated exertion of cognitive control.

The AG region also demonstrated an invalid cueing amplitude effect, which occurred for both correctly identified old and new items. Thus, a strict dependence upon retrieval is also untenable for its functional characterization. However, the region dissociated from the more dorsal SMG in the resting-state and taskevoked connectivity data (Figs. 3, 4), and was instead linked to a network involving both lateral and medial temporal lobe regions known to support memory retrieval. The close coupling of this region with the anteromedial seed network, particularly when unexpectedly old items are encountered (Fig. 4Ci), suggests that it may be involved in the upregulation of episodic retrieval salience via interaction with lateral and medial temporal lobe regions, particularly when retrieval is unexpected. 
The current findings appear to fit best with the theory of Cabeza et al. (2008) that dorsal and ventral parietal responses track top-down and bottom-up retrieval mechanisms, respectively. However, the current findings also suggest some modifications. First, as previously noted, the current SMG and AG responses are not entirely coincident with the superior and inferior parietal responses linked to attention mechanisms in the visual attention literature, which instead tend to occur in more medial aspects of SPL for top-down attention, and anterior to AG, in SMG/temporoparietal junction (TPJ), for bottom-up attention (Hutchinson et al., 2009). Also, a strict division between SMG and $A G$ in the current findings in terms of top-down versus bottom-up processes is inappropriate since the cueing paradigm suggests that the BOLD amplitude of both regions is heavily governed by cued expectations.

Instead, the functional significance of these two regions appears to be linked to the two different networks with which they communicate once expectations are violated. In this light, the SMG was linked to regions supporting cognitive control in nonmemory domains, whereas the AG was linked to regions supporting semantic and episodic memory. This dissociation occurred despite the fact that both regions behaved similarly in terms of the amplitude of their evoked responses. Under the current account, both SMG and AG responses are not the direct result of successful retrieval, but instead reflect a lack of correspondence between retrieval outcomes and expectations. It therefore follows that damage to these regions should not yield a primary memory deficit, but should instead manifest in impaired sensitivity to violations of memory-linked expectations or the exertion of cognitive control in response to these violations, perhaps particularly when unexpectedly familiar items are encountered in contexts where subjects have a strong a priori expectation of novel stimuli (Fig. 4Ci). In the perceptual domain, recent research suggests that areas such as the AG and the proximal TPJ may contribute to "salience maps" that govern or track the tendency of visual objects to capture attention as a function of observer expectations (McCarthy et al., 1997; Downar et al., 2002; Zenon et al., 2009). If memory systems work in an analogous manner [as has been suggested by Vilberg and Rugg (2009b)], then the salience of mnemonic events may be governed, not solely by the level or nature of recovered evidence, but by the "contrast" between the evidence and that which was anticipated.

A key focus for future research in this area should be on potentially dissociating the detection of violations of mnemonic expectancy from the implementation of cognitive control mechanisms once a violation has been detected. In the current analyses, the examination of invalidly cued correct responses implicates both mechanisms. One tentative hypothesis is that amplitude increase in parietal regions signals the detection of a violation, whereas the pattern of connectivity may instead signal regional interactions necessary for successfully countermanding expectations. Manipulating the onset asynchrony between the cues and memoranda, and the use of electrophysiological methods may help to further test this tentative hypothesis. Interestingly, the current data not only implicate the lateral temporal regions in the invalid cueing effect, but also anterior medial portions of the right temporal lobe including hippocampus proper (Fig. 2 B). Traditionally, hippocampal BOLD responses have been considered markers of either successful retrieval for old items, or of increased encoding for novel items. If, however, they are influenced by participant expectations, either directly or through reentrant processes, then their strict interpretation in terms of retrieval or encoding may be questionable.
In summary, we used an episodic analog of a Posner cueing paradigm to alter participants' expectations of subsequent recognition experiences on a trial-by-trial basis. The invalid cueing contrast revealed activation in SMG and AG, parietal regions that have been traditionally associated with retrieval success. These task-evoked invalid cueing responses spatially overlapped with two independently defined resting-state networks identified in the same participants, and a correlation analysis on the taskevoked responses demonstrated the same network selectivity when unexpectedly familiar items were encountered under conditions where the validity of the cues was generally high. Additionally, in an independent dataset, the SMG response was shown to be greater for hits versus correction rejections, but only for observers who generally rarely classified items as old. The response was insensitive to accuracy differences among the observers. These novel findings jointly suggest that the lateral parietal response during recognition is a marker of expectancy violation not a direct reflection of the recovery of episodic content. This interpretation not only brings the data more in line with the vast functional imaging literature on visual search and oddball paradigms, but is also consistent with cognitive models that emphasize the interaction of observer expectations and retrieval outcomes in shaping memory attributions (e.g., Mandler, 1980; Raye and Johnson, 1980; Rajaram, 1993; Benjamin et al., 1998; Whittlesea, 2004; Dobbins and McCarthy, 2008).

\section{References}

Allan K, Rugg MD (1997) An event-related potential study of explicit memory on tests of cued recall and recognition. Neuropsychologia 35:387-397.

Benjamin AS, Bjork RA, Schwartz BL (1998) The mismeasure of memory: when retrieval fluency is misleading as a metamnemonic index. J Exp Psychol Gen 127:55-68.

Botvinick MM, Braver TS, Barch DM, Carter CS, Cohen JD (2001) Conflict monitoring and cognitive control. Psychol Rev 108:624-652.

Braver TS, Bongiolatti SR (2002) The role of frontopolar cortex in subgoal processing during working memory. Neuroimage 15:523-536.

Brett M, Anton J-L, Valabregue R, Poline JB (2002) Region of interest analysis using an SPM toolbox. Paper presented at 8th International Conference on Functional Mapping of the Human Brain, Sendai, Japan, June.

Bunge SA, Wendelken C, Badre D, Wagner AD (2005) Analogical reasoning and prefrontal cortex: evidence for separable retrieval and integration mechanisms. Cereb Cortex 15:239-249.

Cabeza R, Ciaramelli E, Olson IR, Moscovitch M (2008) The parietal cortex and episodic memory: an attentional account. Nat Rev Neurosci 9:613-625.

Christoff K, Gabrieli JDE (2000) The frontopolar cortex and human cognition: evidence for a rostrocaudal hierarchical organization within the human prefrontal cortex. Psychobiology 28:168-186.

Corbetta M, Shulman GL (2002) Control of goal-directed and stimulusdriven attention in the brain. Nat Rev Neurosci 3:201-215.

Corbetta M, Patel G, Shulman GL (2008) The reorienting system of the human brain: from environment to theory of mind. Neuron 58:306-324.

Daselaar SM, Fleck MS, Cabeza R (2006) Triple dissociation in the medial temporal lobes: recollection, familiarity, and novelty. J Neurophysiol 96:1902-1911.

Davidson PSR, Anaki D, Ciaramelli E, Cohn M, Kim ASN, Murphy KJ, Troyer AK, Moscovitch M, Levine B (2008) Does lateral parietal cortex support episodic memory? Evidence from focal lesion patients. Neuropsychologia 46:1743-1755.

Dobbins IG, McCarthy D (2008) Cue-framing effects in source remembering: a memory misattribution model. Mem Cognit 36:104-118.

Dobbins IG, Foley H, Schacter DL, Wagner AD (2002) Executive control during episodic retrieval: multiple prefrontal processes subserve source memory. Neuron 35:989-996.

Dobbins IG, Rice HJ, Wagner AD, Schacter DL (2003) Memory orientation and success: separable neurocognitive components underlying episodic recognition. Neuropsychologia 41:318-333.

Downar J, Crawley AP, Mikulis DJ, Davis KD (2002) A cortical network 
sensitive to stimulus salience in a neutral behavioral context across multiple sensory modalities. J Neurophysiol 87:615-620.

Henson RNA, Rugg MD, Shallice T, Josephs O, Dolan RJ (1999) Recollection and familiarity in recognition memory: an event-related functional magnetic resonance imaging study. J Neurosci 19:3962-3972.

Herron JE, Henson RNA, Rugg MD (2004) Probability effects on the neural correlates of retrieval success: an fMRI study. Neuroimage 21:302-310.

Horovitz SG, Skudlarski P, Gore JC (2002) Correlations and dissociations between BOLD signal and P300 amplitude in an auditory oddball task: a parametric approach to combining fMRI and ERP. Magn Reson Imaging 20:319-325.

Hutchinson JB, Uncapher MR, Wagner AD (2009) Posterior parietal cortex and episodic retrieval: convergent and divergent effects of attention and memory. Learn Mem 16:343-356.

Konishi S, Wheeler ME, Donaldson DI, Buckner RL (2000) Neural correlates of episodic retrieval success. Neuroimage 12:276-286.

Kouneiher F, Charron S, Koechlin E (2009) Motivation and cognitive control in the human prefrontal cortex. Nat Neurosci 12:939-945.

Linden DEJ, Prvulovic D, Formisano E, Völlinger M, Zanella FE, Goebel R, Dierks T (1999) The functional neuroanatomy of target detection: an fMRI study of visual and auditory oddball tasks. Cereb Cortex 9:815-823.

Lyle KB, Johnson MK (2007) Source misattributions may increase the accuracy of source judgments. Mem Cognit 35:1024-1033.

Mandler G (1980) Recognizing: the judgment of previous occurrence. Psychol Rev 87:252-271.

Mather M, Henkel LA, Johnson MK (1997) Evaluating characteristics of false memories: remember/know judgments and memory characteristics questionnaire compared. Mem Cognit 25:826-837.

McCarthy G, Luby M, Gore J, Goldman-Rakic P (1997) Infrequent events transiently activate human prefrontal and parietal cortex as measured by functional MRI. J Neurophysiol 77:1630-1634.

McDermott KB, Jones TC, Petersen SE, Lageman SK, Roediger HL 3rd (2000) Retrieval success is accompanied by enhanced activation in anterior prefrontal cortex during recognition memory: an event-related fMRI study. J Cogn Neurosci 12:965-976.

Poldrack RA (2006) Can cognitive processes be inferred from neuroimaging data? Trends Cogn Sci 10:59-63.

Posner MI, Snyder CRR, Davidson BJ (1980) Attention and the detection of signals. J Exp Psychol Gen 109:160-174.

Rajaram S (1993) Remembering and knowing: two means of access to the personal past. Mem Cognit 21:89-102.

Raye CL, Johnson MK (1980) Reality monitoring vs. discriminating between external sources of memories. Bull Psychon Soc 15:405-408.
Rugg MD, Doyle MC (1992) Event-related potentials and recognition memory for low- and high-frequency words. J Cogn Neurosci 4:69-79.

Shannon BJ, Buckner RL (2004) Functional-anatomic correlates of memory retrieval that suggest nontraditional processing roles for multiple distinct regions within posterior parietal cortex. J Neurosci 24:10084-10092.

Simons JS, Peers PV, Hwang DY, Ally BA, Fletcher PC, Budson AE (2008) Is the parietal lobe necessary for recollection in humans? Neuropsychologia 46:1185-1191.

Simons JS, Peers PV, Mazuz YS, Berryhill ME, Olson IR (2010) Dissociation between memory accuracy and memory confidence following bilateral parietal lesions. Cereb Cortex 20:479-485.

Stevens AA, Skudlarski P, Gatenby JC, Gore JC (2000) Event-related fMRI of auditory and visual oddball tasks. Magn Reson Imaging 18:495-502.

Vilberg KL, Rugg MD (2008) Memory retrieval and the parietal cortex: A review of evidence from a dual-process perspective. Neuropsychologia 46:1787-1799.

Vilberg KL, Rugg MD (2009a) Functional significance of retrieval-related activity in lateral parietal cortex: evidence from fMRI and ERPs. Hum Brain Mapp 30:1490-1501.

Vilberg KL, Rugg MD (2009b) An investigation of the effects of relative probability of old and new test items on the neural correlates of successful and unsuccessful source memory. Neuroimage 45:562-571.

Vincent JL, Snyder AZ, Fox MD, Shannon BJ, Andrews JR, Raichle ME, Buckner RL (2006) Coherent spontaneous activity identifies a hippocampal-parietal memory network. J Neurophysiol 96:3517-3531.

Vincent JL, Kahn I, Snyder AZ, Raichle ME, Buckner RL (2008) Evidence for a frontoparietal control system revealed by intrinsic functional connectivity. J Neurophysiol 100:3328-3342.

Wager TD, Nichols TE (2003) Optimization of experimental design in fMRI: a general framework using a genetic algorithm. Neuroimage 18:293-309.

Wagner AD, Shannon BJ, Kahn I, Buckner RL (2005) Parietal lobe contributions to episodic memory retrieval. Trends Cogn Sci 9:445-453.

Whittlesea BWA (2004) The perception of integrality: remembering through the validation of expectation. J Exp Psychol Learn Mem Cogn 30:891-908.

Wilding EL (2000) In what way does the parietal ERP old/new effect index recollection? Int J Psychophysiol 35:81-87.

Yonelinas AP, Otten LJ, Shaw KN, Rugg MD (2005) Separating the brain regions involved in recollection and familiarity in recognition memory. J Neurosci 25:3002-3008.

Zenon A, Filali N, Duhamel JR, Olivier E (2009) Salience representation in the parietal and frontal cortex. J Cogn Neurosci. Advance online publication. Retrieved August 20, 2009. doi:10.1162/jocn.2009.21233. 